\title{
ARTICLE Microfold cell-dependent antigen transport alleviates infectious colitis by inducing antigen-specific cellular immunity
}

Yutaka Nakamura ${ }^{1,2}$, Hitomi Mimuro ${ }^{3,4}$, Jun Kunisawa ${ }^{5,6}$, Yukihiro Furusawa ${ }^{1,11}$, Daisuke Takahashi ${ }^{1}$, Yumiko Fujimura ${ }^{1}$, Tsuneyasu Kaisho ${ }^{7}$, Hiroshi Kiyono ${ }^{5,8,9,10}$ and Koji Hase ${ }^{1,5}$

Infectious colitis is one of the most common health issues worldwide. Microfold (M) cells actively transport luminal antigens to gutassociated lymphoid tissue to induce $\lg A$ responses; however, it remains unknown whether $M$ cells contribute to the induction of cellular immune responses. Here we report that $\mathrm{M}$ cell-dependent antigen transport plays a critical role in the induction of Th1, Th17, and Th22 responses against gut commensals in the steady state. The establishment of commensal-specific cellular immunity was a prerequisite for preventing bacterial dissemination during enteropathogenic Citrobacter rodentium infection. Therefore, $M$ cell-null mice developed severe colitis with increased bacterial dissemination. This abnormality was associated with mucosal barrier dysfunction. These observations suggest that antigen transport by $\mathrm{M}$ cells may help maintain gut immune homeostasis by eliciting antigen-specific cellular immune responses.

Mucosal Immunology (2020) 13:679-690; https://doi.org/10.1038/s41385-020-0263-0

\section{INTRODUCTION}

The gastrointestinal tract is continuously exposed to numerous microbes, including commensal and foodborne pathogens. Pathogenic bacteria like enteropathogenic Escherichia coli (EPEC), Vibrio cholerae, and Shigella often disrupt the epithelial integrity and increase intestinal permeability, causing lethal diarrhea (especially in infancy). Diarrheal diseases caused by enteric infection represent a global health concern, particularly in developing countries. ${ }^{1-3}$ Immunosurveillance in the gut mucosa protects the host against the pathogenic infection and regulates the microbial composition of commensal microbiota. ${ }^{4}$ To conduct immunosurveillance efficiently, exogenous antigens must be internalized into gut-associated lymphoid tissues (GALTs) and/or mesenteric lymph nodes ( $\mathrm{mLNs}$ ) across the epithelial barrier. Specialized epithelial microfold (M) cells localized in the follicleassociated epithelium play a pivotal role in internalizing luminal antigens into GALTs. ${ }^{5,6} \mathrm{M}$ cells actively transcytose a wide range of substances, such as soluble antigens and microbes, by fluid-phase pinocytosis and receptor-mediated endocytosis. M cell-dependent antigen uptake is at least partly mediated by specific receptors such as $\beta_{1}$-integrin, cellular prion protein, and glycoprotein-2 (GP2). ${ }^{7}$ Previously, we reported that GP2-dependent bacterial uptake initiates the production of antigen-specific secretory immunoglobulin $A(S-\lg A)$ and serum immunoglobulin $G(\operatorname{lgG}){ }^{8}$
Furthermore, GP2-targeting mucosal vaccines successfully enhanced antigen-specific IgA responses to Salmonella enterica serovar Typhimurium. ${ }^{9}$ In addition, GP2-dependent endocytosis facilitated systemic translocation of botulinum toxins with a hemagglutinin domain, thereby enhancing mortality in mice. ${ }^{10}$ Furthermore, various enteropathogenic agents, namely, Shigella spp., Yersinia enterocolitica, Brucella abortus, reovirus, and scrapie prion protein, exploit $M$ cells as a portal of initial invasion. ${ }^{11-15}$ These facts illustrate that $M$ cell-dependent antigen uptake has beneficial and harmful effects in the context of mucosal infection and host defense. Nevertheless, this antigen-sampling pathway is evolutionally conserved from fishes to mammals, ${ }^{16}$ providing the perspective that mucosal immunosurveillance by $M$ cells has conferred advantages for vertebrates.

It also remains obscure whether $M$ cell-dependent antigen uptake contributes to antigen-specific cellular immunity, such as T helper 1 (Th1) and Th17 responses to mucosal pathogens. In addition, it is unknown whether $M$ cell-dependent antigen uptake contributes to immunologic responses against non-invasive mucosal pathogens such as Citrobacter rodentium. This mucosal pathogen causes attaching-effacing lesions and acute bloody diarrhea, which are similar to symptoms observed in human EPEC infection. ${ }^{17}$ A recent study demonstrated that lgG binds to virulence factors on $C$. rodentium to facilitate pathological

\footnotetext{
'Division of Biochemistry, Faculty of Pharmacy and Graduate School of Pharmaceutical Science, Keio University, Tokyo 105-0011, Japan; ${ }^{2}$ Graduate School of Medicine, The University of Tokyo, Tokyo 113-0033, Japan; ${ }^{3}$ Division of Bacteriology, International Research Center for Infectious Diseases, The Institute of Medical Science, The University of Tokyo (IMSUT), 108-8639 Tokyo, Japan; ${ }^{4}$ Division of Infectious Diseases, Research Institute of Microbial Diseases (RIMD), Osaka University, Osaka 565-0871, Japan; ${ }^{5}$ International Research and Development Center for Mucosal Vaccine, The Institute of Medical Science, The University of Tokyo (IMSUT), 108-8639 Tokyo, Japan; ${ }^{6}$ Laboratory of Vaccine Materials, Center for Vaccine and Adjuvant Research and Laboratory of Gut Environmental System, National Institutes of Biomedical Innovation, Health and Nutrition (NIBIOHN), Osaka 567-0085, Japan; ${ }^{7}$ Department of Immunology, Institute of Advanced Medicine, Wakayama Medical University, Wakayama $641-8509$, Japan; ${ }^{8}$ Department of Mucosal Immunology, The University of Tokyo Distinguished Professor Unit, International Research Center for Infectious Diseases, The Institute of Medical Science, The University of Tokyo (IMSUT), Tokyo 1088639, Japan; ${ }^{9}$ Division of Gastroenterology, Department of Medicine, School of Medicine and Chiba University-UCSD Center for Mucosal Immunology, Allergy and Vaccines (cMAV), University of California, San Diego, CA 92093, USA and ${ }^{10}$ Department of Immunology, Graduate School of Medicine, Chiba University, Chiba 260-0856, Japan

Correspondence: Koji Hase (hase-kj@pha.keio.ac.jp)

${ }^{11}$ Present address: Department of Liberal Arts and Sciences, Toyama Prefectural University, Toyama 939-0398, Japan
}

Received: 8 June 2019 Revised: 31 December 2019 Accepted: 13 January 2020

Published online: 10 February 2020 
eradication from the epithelial layer. ${ }^{18}$ Furthermore, antigenspecific $\mathrm{CD}^{+} \mathrm{T}$ cells also critically contribute to host defense against $C$. rodentium infection by enhancing mucosal barrier functions. ${ }^{19-21}$ In particular, T-bet ${ }^{+}$interleukin (IL)-22-producing Th cells were found to play a protective role against lethal infection with high-dose $C$. rodentium. ${ }^{22}$ Correspondingly, ablation of epithelial IL-22 signaling attenuated mucosal barrier functions and eventually caused systemic dissemination of commensal bacteria, leading to lethal systemic inflammation. ${ }^{23}$ These observations raise the possibility that blocking the systemic dissemination of commensals by antigen-specific cellular immune responses may be essential for ameliorating the pathology of $C$. rodentium.

Here we addressed the contribution of M-cell-dependent antigen transport to host defense and maintaining immunological homeostasis during $\mathrm{C}$. rodentium infection, using $\mathrm{Spib}^{-1-}$ mice lacking mature $\mathrm{M}$ cells. ${ }^{24}$ We found that the absence of $\mathrm{M}$ cells did not affect $C$. rodentium eradication on the mucosa; however, it exacerbated systemic dissemination of commensal bacteria due to attenuated commensal-specific Th1 and Th17 responses, resulting in the development of chronic and severe colitis. Based on these observations, $M$ cell-dependent antigen uptake was deemed essential for the fitness of the gut mucosal barrier system by inducing antigen-specific immunity to gut microbes.

\section{RESULTS}

$M$ cells mitigated infectious colitis by preventing systemic bacterial translocation

To explore the role of $M$ cells in host defense against mucosal infection, we infected mature $M$ cell-null Spib $^{-/-}$mice $^{24}$ with $C$. rodentium, a potent inducer of both cellular (Th1 and Th17) and humoral $(\operatorname{lgA})$ immune responses in the gut. ${ }^{25,26}$ We found that $\mathrm{Spib}^{-1-}$ mice, but not co-housed wild-type (WT) mice, showed a wasting-like disease with approximately $15 \%$ loss of initial body weight at 14 days postinfection (dpi) (Fig. 1a). This pathogenic symptom was associated with increased clinical scores (i.e., bloody feces and diarrhea) owing to chronic and severe bloody diarrhea (Fig. 1 b). Furthermore, the number of $C$. rodentium in the feces of $\mathrm{Spib}^{-1-}$ mice was significantly higher than that of WT mice during infection. Although the pathogen was nearly eliminated in both groups at $16 \mathrm{dpi}$ (Fig. 1c), histological abnormalities such as substantial edema, massive myeloid cell infiltration, and loss of epithelial integrity persisted in the colon of Spib ${ }^{-/-}$mice (Fig. 1d and Supplementary Fig. 1a), indicative of severe colitis. Flow cytometric analysis also confirmed that myeloid cell migration increased in the colonic tissue (Supplementary Fig. 1a-c). In addition, $\mathrm{Spib}^{-1-}$ mice displayed splenomegaly from $7 \mathrm{dpi}$ onwards (Fig. 1e, f). The presence of splenomegaly could reflect bacterial translocation from the gut lumen. Indeed, substantially more aerobic and anaerobic bacteria were found in the spleens of $\mathrm{Spib}^{-1-}$ mice than in WT mice at $12 \mathrm{dpi}$, when splenomegaly peaked (Fig. 1g). Likewise, the translocation of anaerobic bacteria to $\mathrm{mLNs}$ tended to increase in $\mathrm{Spib}^{-/-}$mice (Supplementary Fig. 1d). In contrast, there were no significant differences in the translocation of $C$. rodentium to the spleen (Supplementary Fig. 1e). These observations suggest that $M$ cell-dependent antigen uptake is most likely required for establishing hostdefense functions that protect against bacterial translocation and limit colonic inflammation during mucosal infection.

Spi-B is expressed by $M$ cells and several hematopoietic cell lineages, including plasmacytoid dendritic cells (DCs) and B cells, ${ }^{27,28}$ and also regulates myeloid differentiation. ${ }^{29}$ To determine whether attenuation of the mucosal-protective function in $\mathrm{Spib}^{-1-}$ mice was attributable to the absence of $\mathrm{M}$ cells, we generated mixed bone marrow (BM) chimeric C57BL/6 mice harboring either Spi-B-deficient or WT hematopoietic cells. Upon infection, the clinical scores and extent of body weight loss were comparable between the two groups (Fig. 2a, b). Likewise, inflammatory symptoms in the colon were only marginally observed in both groups (Fig. 2c). In contrast, Spib ${ }^{-/-}$mice transplanted with WT hematopoietic cells showed recapitulation of the phenotypes of conventional Spib ${ }^{-1-}$ mice after $C$. rodentium infection, as evidenced by severe body weight loss and high clinical scores (Fig. 2d, e). The inflammatory symptoms were also markedly exacerbated in mice lacking non-hematopoietic Spi-B compared to Spi-B-sufficient mice (Fig. 2f). These data demonstrated that Spi-B expression in hematopoietic cell lineages conferred little, if any, protection against $C$. rodentium infection.

$M$ cells were dispensable for pathogen-specific cellular immunity in the effector site. We further sought to elucidate the mechanism whereby the $M$ cell-dependent immune response protects against infectious colitis and systemic bacterial translocation. The disturbance of intestinal microbiota, termed dysbiosis, has been implicated in the development of inflammatory bowel diseases. We observed that the bacterial compositions were comparable between $\mathrm{Spib}^{-/-}$mice and co-housed WT mice (Supplementary Fig. 2), indicative of normal gut microbiota in the absence of $M$ cells.

A recent study demonstrated that serum lgG specific for $C$. rodentium played a critical role in eliminating $C$. rodentium from the epithelial layer. ${ }^{18}$ However, we readily detected the same level of C. rodentium-specific IgG in Spib ${ }^{-1-}$ and WT mice at $16 \mathrm{dpi}$ (Supplementary Fig. 3a). Thus GALTs appeared to be dispensable for the $C$. rodentium-specific IgG response. The regular eradication of C. rodentium in $\mathrm{Spib}^{-1-}$ mice at 16 dpi supported this view (Fig. 1c). C. rodentium-specific IgA in feces was only induced in WT mice at the terminal stage ( $16 \mathrm{dpi}$; Supplementary Fig. $3 \mathrm{~b}$ ). Thus the dependency upon $M$ cells was different between IgA and IgG responses to $C$. rodentium infection. Even though the antigenspecific S-IgA response was severely affected in the absence of $M$ cells, S-IgA may play a minimal role in eradicating $C$. rodentium due to the lack of a response at the middle stage ( $7 \mathrm{dpi}$; Supplementary Fig. 3b).

We subsequently interrogated cellular immune responses to $C$. rodentium infection. In WT mice, the number of $C$. rodentiumspecific Th1, Th17, and Th22 cells in Peyer's patches (PPs) was highly elevated at the late stages (12 and $16 \mathrm{dpi}$; Fig. 3a-c). However, such cellular immune responses against $C$. rodentium were only weakly induced in PPs of Spib ${ }^{-1-}$ mice. Similar trends were observed in cecal patches (Supplementary Fig. 4a). Nevertheless, the number of $C$. rodentium-specific Th cell populations in the effector site, namely, the colonic lamina propria (cLP), tended to increase or remained unchanged in $\mathrm{Spib}^{-/-}$mice at $16 \mathrm{dpi}$ (Fig. $3 \mathrm{~d}-\mathrm{f}$ ). These facts imply that the vast majority of $C$. rodentiumspecific Th cells at the terminal stage of infection were locally generated in the colon or derived from secondary lymphoid tissues other than GALTs. In support of this possibility, $C$. rodentium-specific Th cells also concurrently increased in the spleen of $\mathrm{Spib}^{-1-}$ mice (Supplementary Fig. 4b), indicating that early bacterial translocation in $\mathrm{Spib}^{-1-}$ mice promoted the generation of $C$. rodentium-specific Th cells in the colon and/or systemic lymphoid tissues, like the spleen. Thus we considered that the increases of $C$. rodentium-specific Th cells observed in the $\mathrm{CLP}$ of $\mathrm{Spib}^{-/-}$mice during the later phase of infection were secondary effects resulting from bacterial translocation.

$M$ cell-dependent antigen transport facilitated the cellular immune response to gut commensals. Mucosal infection frequently causes epithelial barrier dysfunction, leading to systemic translocation of commensal bacteria. ${ }^{23}$ Preexisting commensal-specific memory lymphocytes minimize such bacterial leakage. Notably, we observed significant decreases in commensal-specific Th1, Th17, and Th22 cells in the CLP of Spib ${ }^{-/-}$mice before C. rodentium infection ( $0 \mathrm{dpi}$; Fig. $4 \mathrm{a}-\mathrm{c}$ ). This trend persisted during the early and middle phases of $C$. rodentium infection ( 7 and 12 dpi; 

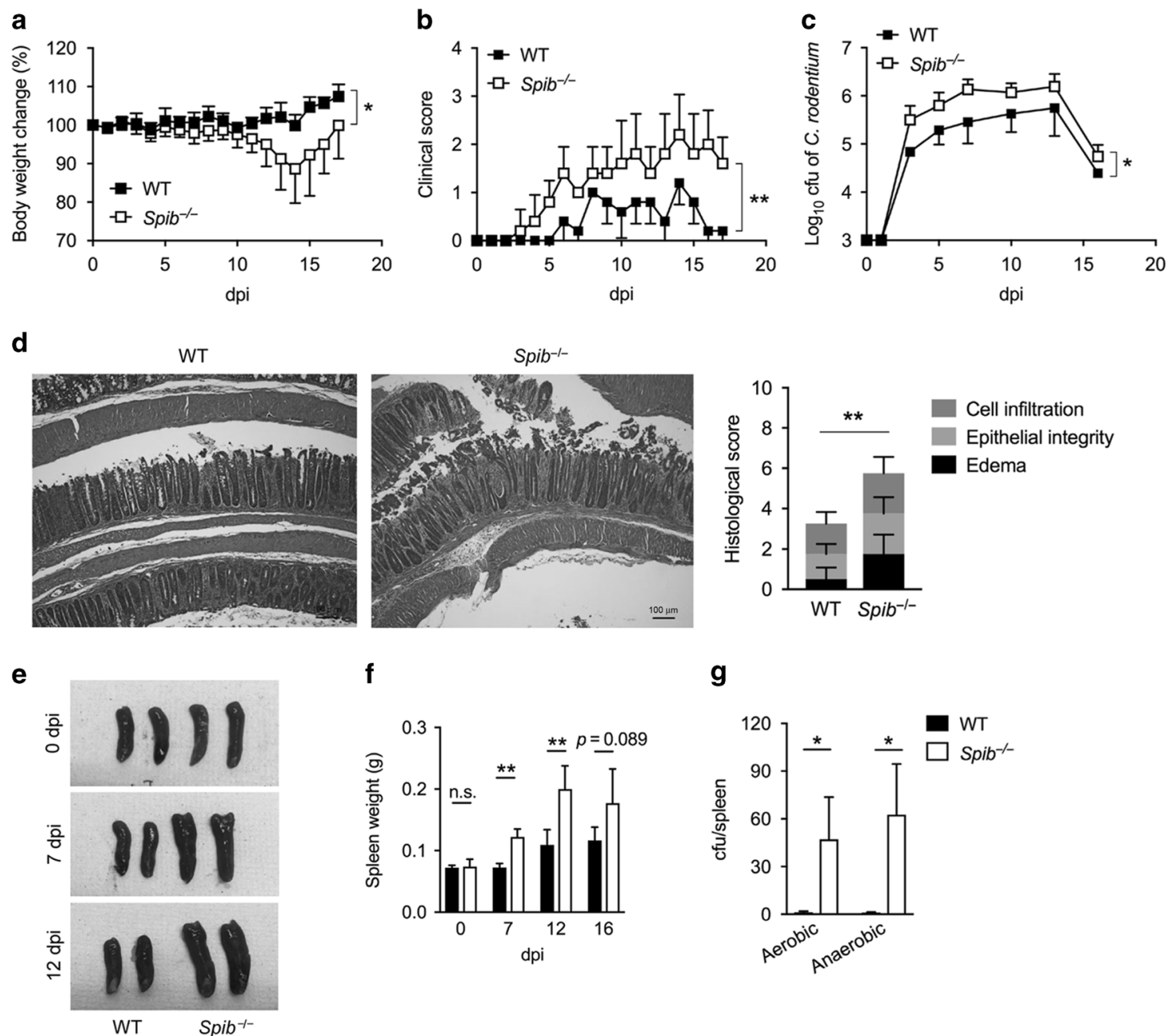

\section{f}

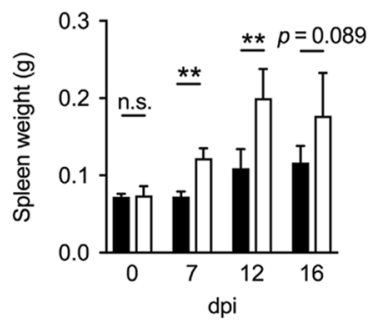

g

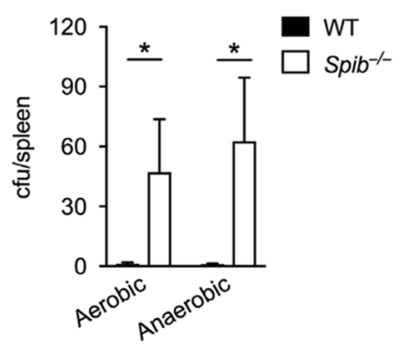

Fig. 1 Susceptibility of Spib ${ }^{-1-}$ mice to C. rodentium-induced infectious colitis. a-g WT and Spib ${ }^{-/-}$mice were orally infected with $1 \times 10^{9}$ cfu of $C$. rodentium. The body weight (a), fecal clinical score (b), and fecal cfu of $C$. rodentium (c) were measured every day during the experimental period in WT and Spib ${ }^{-1-}$ mice. d Colonic sections at $16 \mathrm{dpi}$ were stained with hematoxylin and eosin for histological examination. Scale bars, $100 \mu \mathrm{m}$. e-g Spleens were observed at 0, 7, and 12 dpi (e). f Spleen weights were measured in WT and Spib ${ }^{-/-}$mice at 0, 7, 12, and 16 dpi $(n=$ 4/group for each time point). The values are shown as the mean \pm SD $(n=4)$. The data shown are representative of at least two independent experiments with similar results. g Aerobic and anaerobic bacteria translocating into spleen were detected in WT and Spib ${ }^{-/-}$mice at 12 dpi ( $n=9$ and 8 , respectively). The values are shown as the mean \pm SEM of two independent experiments. ${ }^{*} p<0.05,{ }^{* *} p<0.01$, n.s.: not significant by repeated ANOVA testing $(\mathbf{a}-\mathbf{c})$, Student's $t$ test, Welch's $t$ test $(\mathbf{d}, \mathbf{f})$, or Mann-Whitney $U$ test $(\mathbf{g})$.

Fig. 4a-c). However, no difference was found in the total numbers of Th1 and Th17 cells between WT and Spib ${ }^{-1-}$ mice at steady state (Supplementary Fig. 5a-c), because the commensal-specific Th cell subsets are relatively minor populations among the effector Th cell subsets. The reduction of commensal-specific Th cells in the effector site was attributed to impaired induction of mucosal immune responses in GALTs, where both total and commensalspecific Th1, Th22, and Th17 cells were severely affected in the absence of $M$ cells in the steady state (0 dpi; Fig. $4 d-f$, Supplementary Fig. $5 \mathrm{~d}-\mathrm{f}$ ). Spib ${ }^{-1-}$ mice transplanted with WT hematopoietic cells also showed similar trends of attenuated commensal-specific Th cell responses in PPs (Supplementary Fig. 6a-d). Besides, this trend was still observed after mucosal infection (7-16 dpi; Fig. $4 d-f$ ). It is therefore conceivable that the failure of the commensal-specific cellular immune response in the inductive site resulted in fewer commensal-specific Th cells in the effector site (Fig. $4 a-c)$. Together, these data illustrate that the loss of $\mathrm{M}$ cell-dependent antigen uptake attenuated the induction of commensal-specific immune responses, possibly leading to mucosal barrier dysfunctions, thereby enhancing systemic translocation of luminal microbes during mucosal invasion.
$M$ cell-dependent antigen transport was required for the establishment of memory $T$ cells. Upon activation, naive $T$ cells differentiate into central memory $\mathrm{T}$ cells $\left(\mathrm{T}_{\mathrm{CM}} ; \mathrm{CD} 44^{+} \mathrm{CD} 62 \mathrm{~L}^{\text {high }}\right.$ $\left.\mathrm{CD} 69^{-/ \text {low }}\right)$ and resident memory $T$ cells $\left(T_{R M} ; C D 44^{+} C D 62 L^{i n t}\right.$ CD69 ${ }^{\text {high }}$ ) cells. While $T_{C M}$ cells are mainly localized to secondary lymphoid tissues, including GALTs, resident memory $T$ cells are distributed to peripheral tissues like the cLP. ${ }^{30}$ Reduced commensal-specific effector $T$ cells due to the absence of mature $M$ cells may result from these memory $T$ cell subsets. The total memory Th cell population decreased by $>50 \%$ in $^{S_{p i b}}{ }^{-/-}$mice versus WT mice (Fig. $4 \mathrm{~g}, \mathrm{~h}$ ). $\mathrm{T}_{\mathrm{CM}}$ cells were severely depleted in $\mathrm{Spib}^{-1-}$ mice (Fig. 4i). Considering that our CLP preparation embraced solitary intestinal lymphoid tissues (SILTs), ${ }^{31}$ this result may reflect fewer $\mathrm{T}_{\mathrm{CM}}$ cells in SILTs of Spib ${ }^{-/-}$mice. The number of $\mathrm{T}_{\mathrm{RM}}$ cells also significantly decreased in Spib ${ }^{-1-}$ mice (Fig. 4j). Together, these data corroborated that M-cell-dependent antigen uptake was indispensable for inducing and/or maintaining memory $\mathrm{CD}^{+}{ }^{+} \mathrm{T}$ cells under physiological conditions.

$M$ cells were essential for GALT maturation. The attenuated immune response to commensals due to the absence of mature 


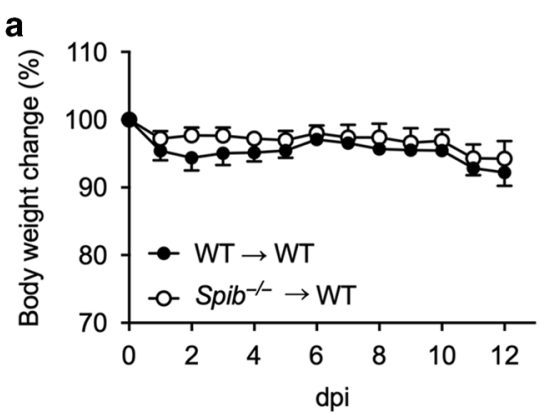

C
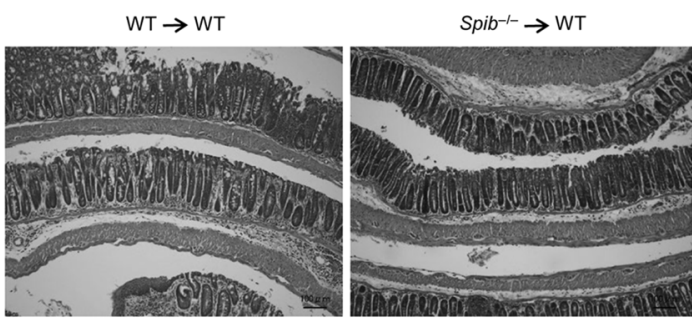

b

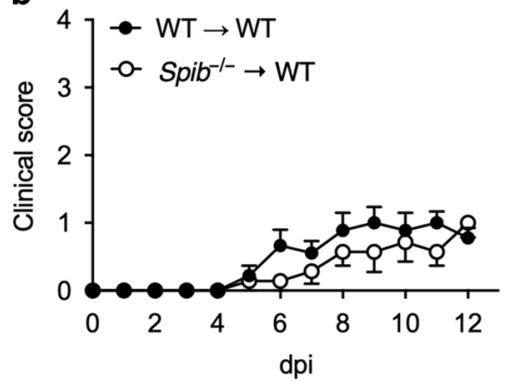

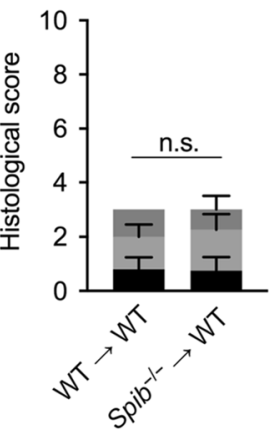

Cell infiltration

Epithelial integrity

Edema

d

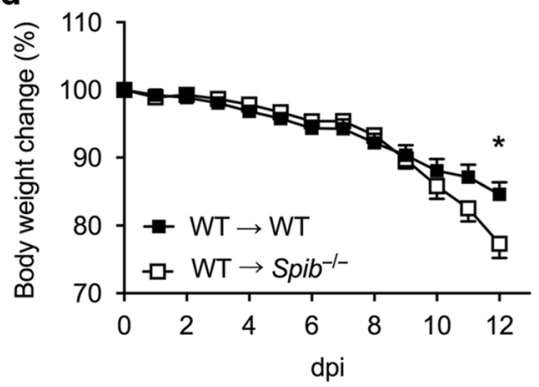

e

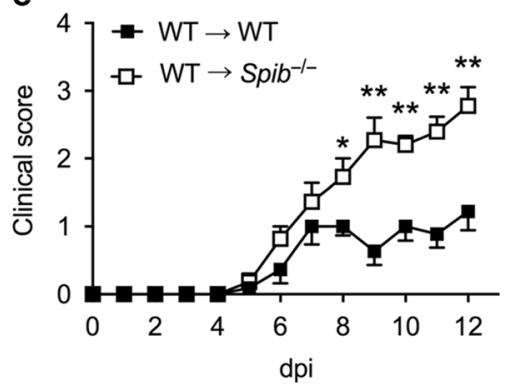

$\mathbf{f}$
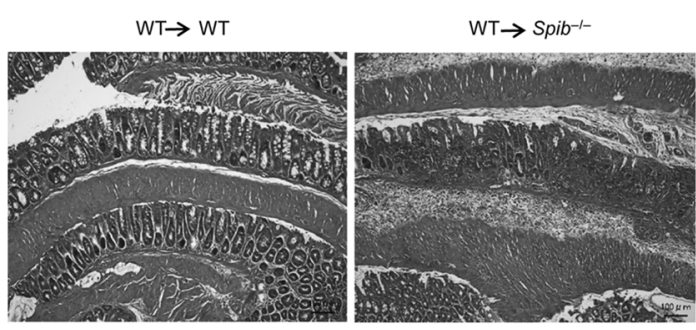

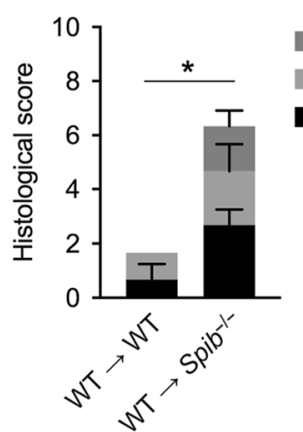

Cell infiltration

Epithelial integrity

Edema

Fig. 2 The deficiency in non-hematopoietic Spi-B exacerbated infectious colitis. a-c Congenic WT recipients with a bone marrow transplant from WT or Spib ${ }^{-/-}$mice were infected with C. rodentium ( $n=9$ and 7, respectively). d-f WT and Spib ${ }^{-1-}$ recipients with a bone marrow transplant from congenic WT mice were infected with $C$. rodentium $(n=11)$. Body weights $(\mathbf{a}$, $\mathbf{d})$ and clinical scores $(\mathbf{b}$, e) were measured every day during the experimental period. Colonic sections at $12 \mathrm{dpi}$ were stained with hematoxylin and eosin for histological examination (c, f). Scale bars: $100 \mu \mathrm{m}$. The values are shown as the mean \pm SEM of two independent experiments. ${ }^{*} p<0.05,{ }^{* *} p<0.01$, n.s.: not significant by Student's $t$ test or Welch's $t$ test.

M cells may have reflected a functional disorder of GALTs. Thus we further analyzed the development and function of GALTs in Spib ${ }^{-/-}$ mice under physiological conditions. PPs of Spib ${ }^{-1-}$ mice were significantly smaller than those of WT mice (Supplementary Fig. 7a), although the number of PPs did not change in Spib ${ }^{-1-}$ mice (6.5 \pm 1.0 and $7.3 \pm 1.5$ in WT and Spib ${ }^{-1-}$ mice, respectively). These observations indicate that $M$ cell-dependent antigen uptake facilitated the maturation, but not formation, of PPs, consistent with the previous observation that PP organogenesis occurs prenatally. ${ }^{32}$
Moreover, Spib ${ }^{-/-}$mice displayed hypoplasia of cecal patches and colonic patches, the organized lymphoid follicles in the cecum and colon, respectively (Supplementary Fig. 7a, b). This abnormality was accompanied by fewer mature SILTs in the colon (Supplementary Fig. 7c). In line with the hypoplasia of GALTs, we found that total $\mathrm{B}$ and $\mathrm{GL} 7^{+} \mathrm{CD} 95^{+}$germinal center (GC) B cells markedly decreased in PPs, cecal patches, and colonic tissue embracing colonic patches and SILTs of Spib ${ }^{-1-}$ mice (Fig. 5a-C, Supplementary Fig. 8a, b). The decreased total B cells and GC B cells were also observed in $\mathrm{Spib}^{-/-}$mice transplanted with WT 
a

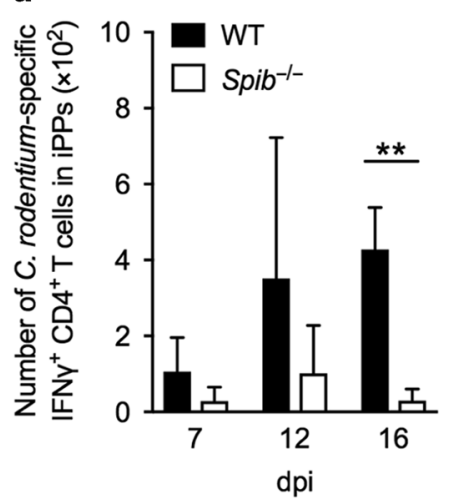

d

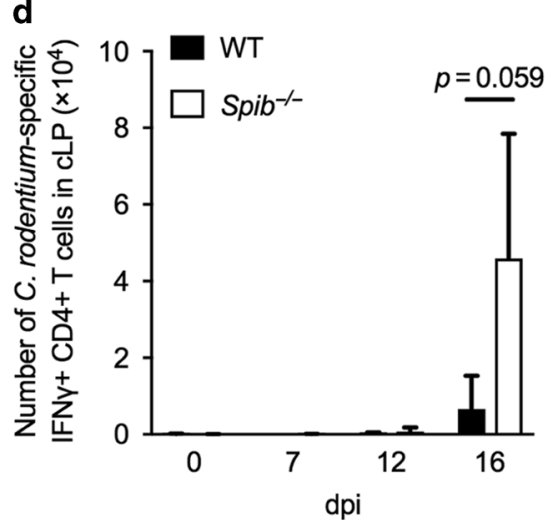

b

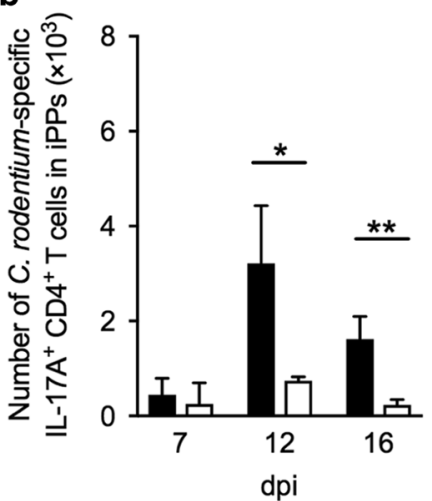

e

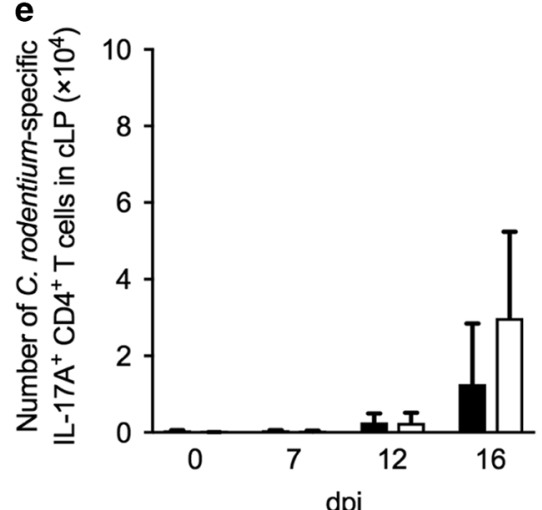

C

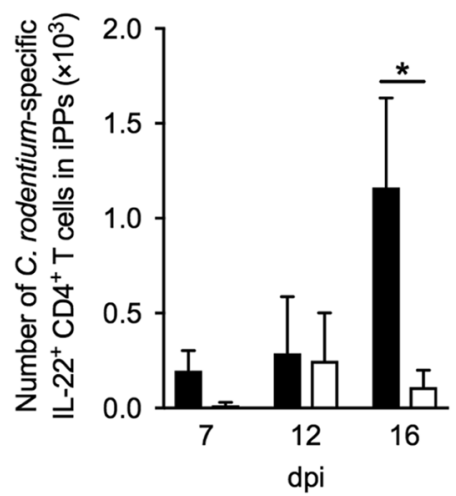

$\mathbf{f}$

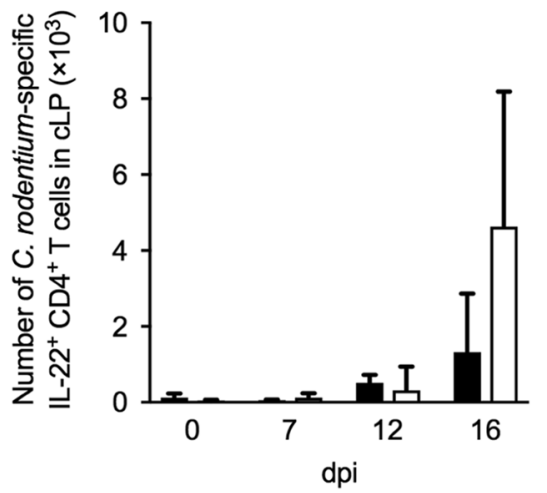

Fig. 3 Commensal-specific, but not pathogen-specific, Th immune responses were compromised in the absence of Spi-B. a-f WT and $\mathrm{Spib}^{-/-}$mice were infected with C. rodentium to analyze pathogen-specific Th1 (a, d), Th17 (b, e), and Th22 (c, f) responses in iPPs (a-c) and the $\operatorname{cLP}(\mathbf{d}-\mathbf{f})$ at the indicated time points. IFN- $\gamma^{+}, \mathrm{IL}-17 \mathrm{~A}^{+}$, and IL-22 $2^{+}$cells gated as $\mathrm{CD} 4^{+} \mathrm{CD} 3^{+}$cells were defined as Th1, Th17, and Th22, respectively. The data are shown as the mean \pm SD $(n=4)$. The results shown are representative of at least two independent experiments with similar results. ${ }^{*} p<0.05 ;{ }^{* *} p<0.01 ;$ n.s., not significant. Student's $t$ test or Welch's $t$ test.

hematopoietic cells (Supplementary Fig. 6e, f). In contrast, no significant difference was found in the number of GC B cells in $\mathrm{mLNs}$ or the spleen (Supplementary Fig. $8 \mathrm{c}$, d), indicating that lymphocytes in these tissues recognize antigens internalized via $M$ cell-independent pathways. We further examined the possibility that the uptake of commensal bacteria-derived antigens via $M$ cells may facilitate maturation of GALTs by treating WT and Spib ${ }^{-/-}$ mice with a cocktail of antibiotics for 4 weeks after weaning to deplete gut commensal bacteria (Supplementary Fig. 9a, b). This treatment prominently decreased the number of total and GC B cells in GALT of WT mice to the level comparable to untreated Spib $^{-1-}$ mice (Supplementary Fig. 9c, d). In contrast, the antibiotic treatment did not significantly alter total and GC B cells in Spib ${ }^{-1-}$ mice. Thus the uptake of commensal-derived antigens by $M$ cells was essential for inducing GC reactions, specifically in GALTs.

Because the GC reaction in GALTs contributes to antigenspecific IgA generation, we analyzed IgA-producing cells in the gut of $\mathrm{Spib}^{-/-}$mice. The number of $\lg \mathrm{A}^{+}$lymphoblasts $\left(\lg \mathrm{A}^{+} \mathrm{B} 22 \mathrm{O}^{+}\right)$ decreased in PPs (Fig. 5d). Nevertheless, the amount of fecal S-IgA in Spib $^{-1-}$ and WT mice was comparable (Supplementary Fig. 8e). Therefore, we inferred that attenuated $\lg A$ responses in $M$ cell-null GALTs might be compensated for by low-affinity, IgA-induced Tindependent class switching in the effector site. Indeed, the frequency of fecal bacteria heavily coated by $\lg \mathrm{A}$ ( $\lg \mathrm{A}^{\text {high }}$ bacteria) significantly decreased in $\mathrm{Spib}^{-1-}$ mice (Fig. 5e). Thus $\mathrm{M}$ cells significantly contributed to the induction of high-affinity $\lg A$. In contrast, the attenuated affinity of IgA by Spi-B deficiency did not affect the abundance of fecal bacteria and the bacterial translocation into gut-draining lymph nodes and spleen under the physiological conditions (Supplementary Fig. 8f-i).
The commensal-specific Th cell response induced by M-celldependent antigen uptake ameliorated dextran sulfate sodium (DSS)-induced colitis. To rigorously confirm the importance of commensal-specific Th responses in suppressing bacterial dissemination and excessive inflammation, we examined the susceptibility of $\mathrm{Spib}^{-/-}$mice to DSS-induced colitis. Although body weight losses were similar between WT and Spib ${ }^{-/-}$mice (Fig. 6a), the fecal clinical score was significantly higher in Spib ${ }^{-1-}$ mice, indicative of severe mucosal damage (Fig. 6b). Correspondingly, Spi-B deficiency tended to worsen colonic inflammation, as evidenced by enhanced epithelial cell shedding and colon shortening (Fig. 6c, d). Splenomegaly was also observed in Spib $\mathrm{S}^{-1-}$ mice (Fig. 6e). These pathological changes in $\mathrm{Spib}^{-1-}$ mice were reminiscent of the phenotype in $C$. rodentium-induced infectious colitis (Fig. 1b, e, f). Thus we reason that M-cell-dependent antigen uptake likely plays a pivotal role in preventing bacterial dissemination and colitis by inducing the commensal-specific Th cell response.

M cell-dependent induction of commensal-specific Th responses accelerates the epithelial turnover during infection

We subsequently explored the mechanism whereby $M$ celldependent induction of commensal-specific Th responses prevents infectious colitis. Among the Th cell subsets, Th17 cells produce IL-22 and IL-17 to upregulate antimicrobial lectins (e.g., Reg $3 b$ and Reg $3 g$ ) and peptides (e.g., Defb3), respectively, in intestinal epithelial cells. ${ }^{33,34}$ However, Spi-B deficiency did not influence the expression of these molecules during infection (Supplementary Fig. 10a). In addition, the expression levels of tight-junction (TJ)-related genes, namely, Tjp1, Ocln, and Cldn3, 

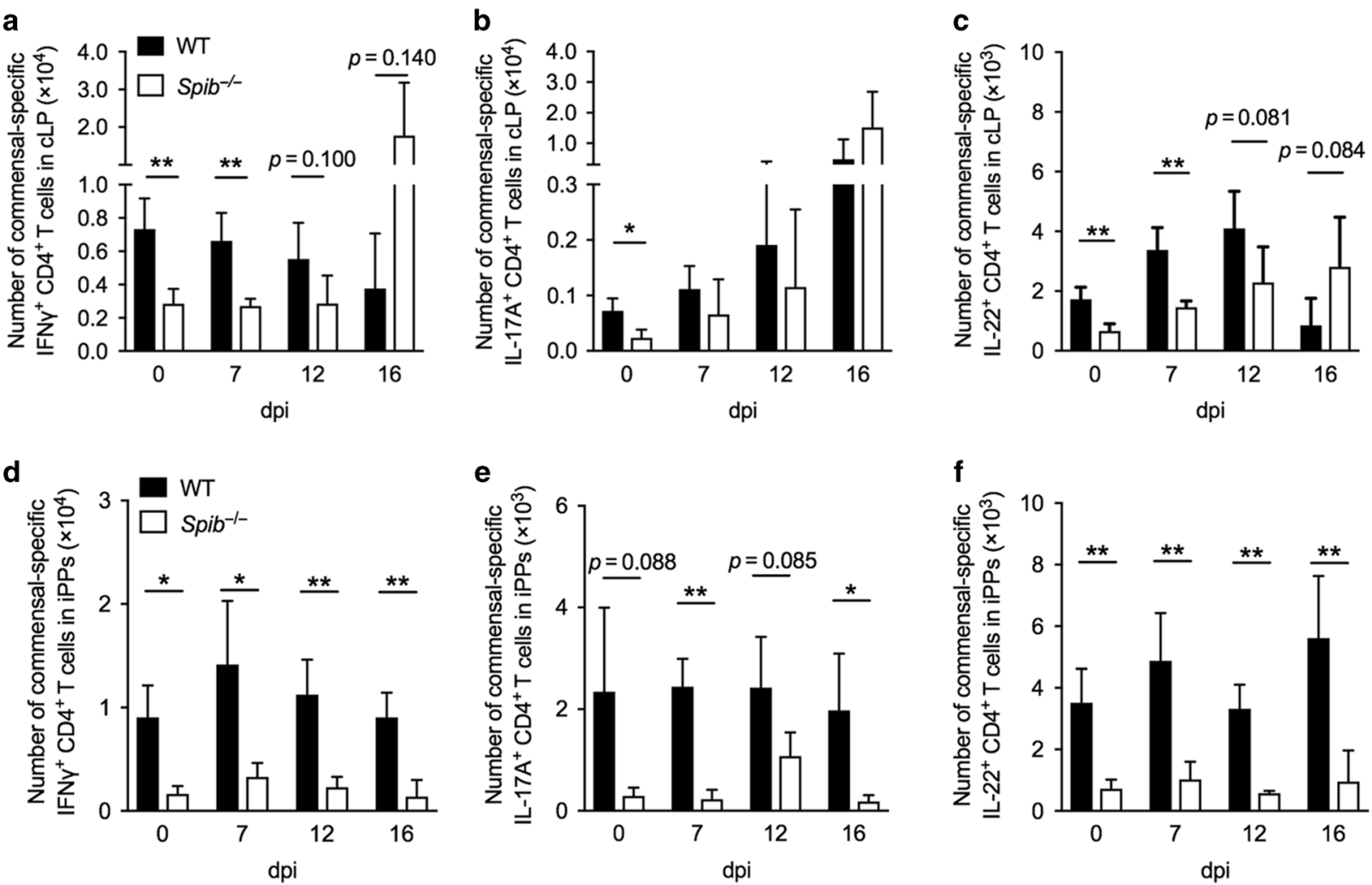

g

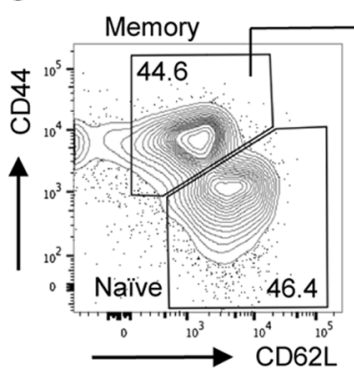

$\mathbf{h}$

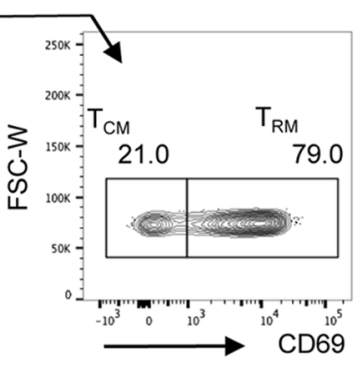

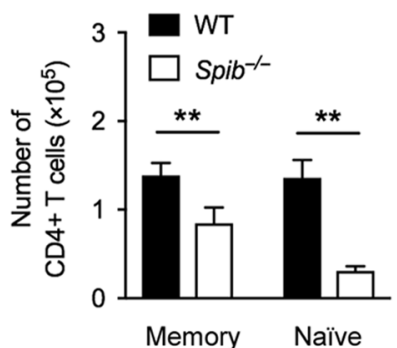
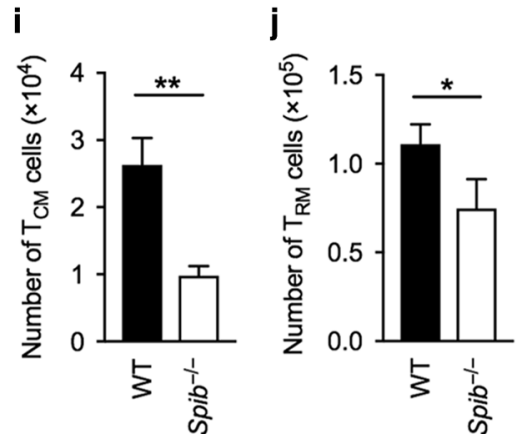

Fig. 4 Attenuated cellular immune responses to commensal microbes in Peyer's patch of $C$. rodentium-infected Spib ${ }^{-/-}$mice. a-f Timecourse analyses of commensal-specific Th1 $(\mathbf{a}, \mathbf{d}), \operatorname{Th} 17(\mathbf{b}, \mathbf{e})$, and Th22 (c, $\mathbf{f})$ cells in $\operatorname{CLP}(\mathbf{a}-\mathbf{c})$ and iPPs (d-f) were performed. g The gating strategy to identify memory $\mathrm{CD}^{+}{ }^{+} \mathrm{T}$ cell subsets is shown. $\mathbf{h}-\mathbf{j}$ The numbers of naive and total memory $\mathrm{CD} 4^{+} \mathrm{T}$ cells $(\mathbf{h}), \mathrm{T}_{\mathrm{CM}}(\mathbf{i})$, and $\mathrm{T}_{\mathrm{RM}}$ cells $(\mathbf{j})$ in the CLP were detected. The data are shown as the mean \pm SD $(n=4,3)$. The results shown are representative of two independent experiments. ${ }^{*} p<0.05,{ }^{* *} p<0.01$, n.s.: not significant by Student's $t$ test or Welch's $t$ test (a-f) and Student's $t$-test (h-j).

was also unchanged (Supplementary Fig. 10b). Moreover, there were no apparent differences in the subcellular localization of $\mathrm{TJ}$ and adherens junction components, such as ZO-1 and E-cadherin, respectively, between WT and $\mathrm{Spib}^{-/-}$mice (Supplementary Fig. 10c).

Colonic epithelial cells vigorously enhance proliferation in response to $C$. rodentium infection, and the enhanced epithelial turnover comprises a fundamental barrier to expelling infected cells. ${ }^{35,36}$ Because Th1-derived interferon (IFN)- $\gamma$ promotes epithelial cell proliferation during $C$. rodentium infection ${ }^{21}$ and the commensal-specific Th1 response was attenuated in Spib ${ }^{-1-}$ mice, we hypothesized that Spi-B deficiency may affect the hyperproliferative response. This hypothesis was tested by performing an in vivo incorporation assay using 5-ethynyl-2' deoxyuridine (EdU). WT and Spib ${ }^{-/-}$mice were treated with EdU at steady or infectious state (at $5 \mathrm{dpi}$ ), and then $\mathrm{EdU}^{+}$epithelial cells were detected at 4 and $48 \mathrm{~h}$ postinjection to dissect proliferating cells and epithelial renewal, respectively (Fig. $7 \mathrm{a}, \mathrm{b}$ ). At steady state, no significant differences in $\mathrm{EdU}^{+}$cells occurred between WT and Spib ${ }^{-/}$mice at 4 and $48 \mathrm{~h}$ postinjection (Fig. 7a). During infection, $\mathrm{EdU}^{+}$ proliferating colonocytes expanded by more than twofold in WT mice (Fig. $7 \mathrm{~b} ; 4 \mathrm{~h}$ postinjection). Nevertheless, $\mathrm{EdU}^{+}$prominently declined at $48 \mathrm{~h}$ postinjection, indicating rapid epithelial turnover in response to mucosal infection. Such hyperproliferative response leading to rapid epithelial turnover were significantly mitigated in $\mathrm{Spib}^{-1-}$ mice (Fig. 7b). To confirm the effects of intestinal CD4 ${ }^{+}$ $\mathrm{T}$ cells on the epithelial turnover, we adoptively transferred intestinal $\mathrm{CD}^{+}$cells of uninfected WT or Spib ${ }^{-1-}$ mice into $\mathrm{Rag}^{1^{-/}}$ recipients before the infection. Intestinal $\mathrm{CD}^{+}$cells from WT accelerated epithelial turnover upon infection, as evidenced by the disappearance of a large part of $\mathrm{EdU}^{+}$cells within $48 \mathrm{~h}$ (Supplementary Fig. 11). However, $\mathrm{CD}^{+}$cells from Spib ${ }^{-1-}$ mice failed to induce such rapid epithelial turnover.

We also examined epithelial turnover in a DSS-induced colitis model. Consistent with the observations in C. rodentium-induced colitis, $\mathrm{EdU}^{+}$proliferating colonocytes significantly decreased in 


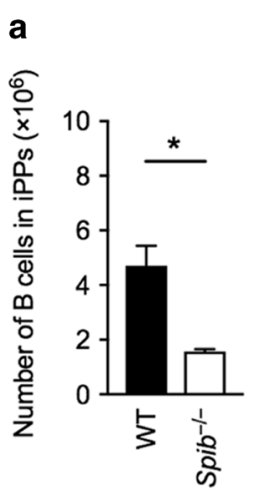

b

d

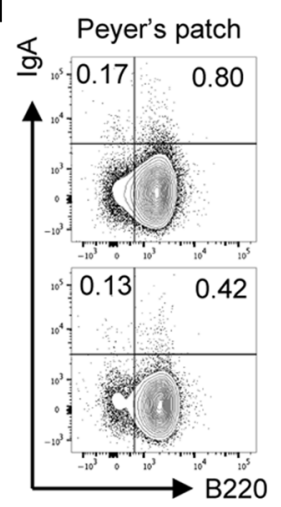

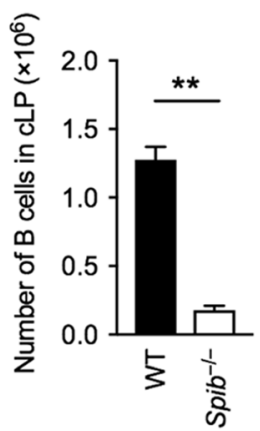

C

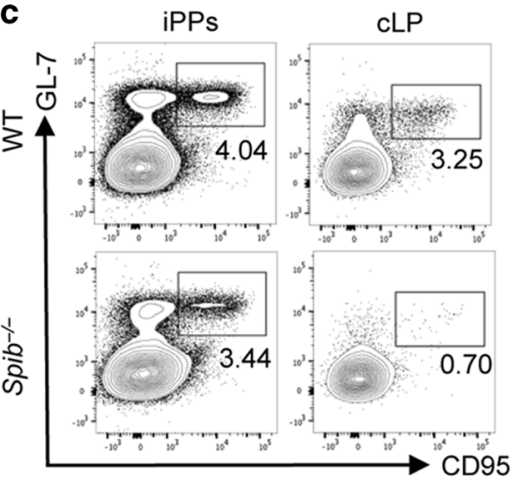

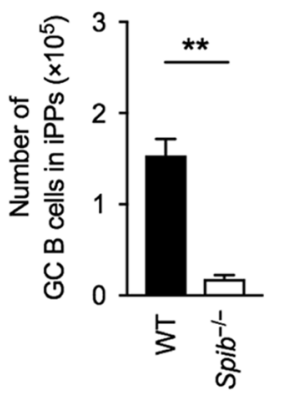

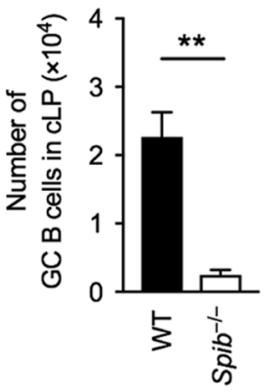

e
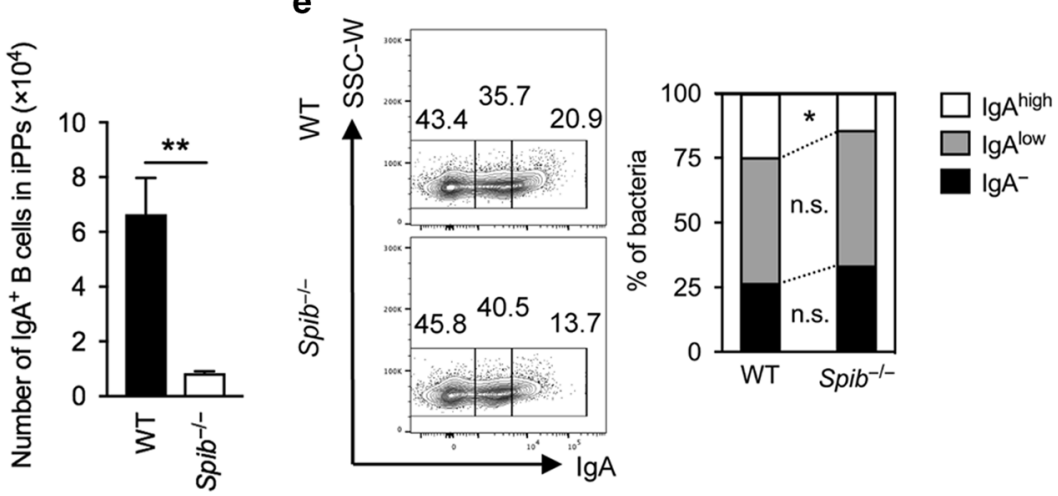

Fig. 5 The reduction of GC reactions and high-affinity IgA in Spib ${ }^{-1-}$ mice in the steady state. $\mathbf{a}, \mathbf{b}$ The total number of B220 $0^{+} \mathrm{CD} 3 \varepsilon^{-}$B cells was assessed in iPPs (a) and the $\operatorname{CLP}(\mathbf{b})$ of WT and $\mathrm{Spib}^{-1-}$ mice in the steady state. The data are shown as the mean \pm SD $(n=4)$ and are representative of two independent experiments. $c$ The numbers of $B 220^{+} \mathrm{CD} 3 \varepsilon^{-} \mathrm{CD} 95^{+} \mathrm{GL}-7^{+} \mathrm{GC} B$ cells were measured in iPPs and the $\mathrm{CLP}$. The data are shown as the mean \pm SEM $(n=7)$ from two independent experiments. $\mathbf{d}$ The numbers of $\lg \mathrm{A}^{+} \mathrm{B} 220^{+} \mathrm{CD} 3 \varepsilon^{-}$lymphoblasts in iPPs were detected. The values are shown as the mean \pm SEM $(n=8)$ from two independent experiments. e Fecal bacteria bound to IgA in WT and $\mathrm{Spib}^{-/-}$mice were analyzed by flow cytometry. The data are shown as the mean \pm SEM $(n=11,10)$ from two independent experiments. ${ }^{*} p<0.05,{ }^{* *} p<0.01$, n.s.: not significant by Student's $t$ test $(\mathbf{a}, \mathbf{b}, \mathbf{d}, \mathbf{e})$ or Welch's $t$ test $(\mathbf{c})$.

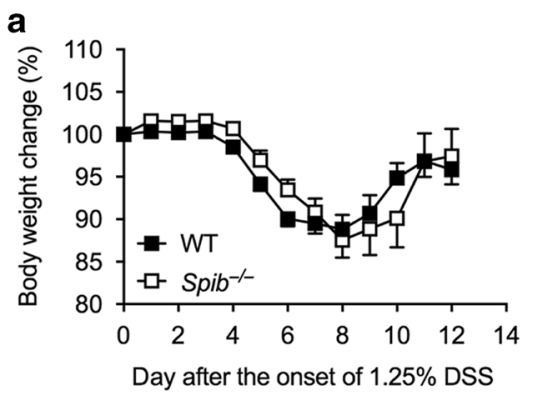

C

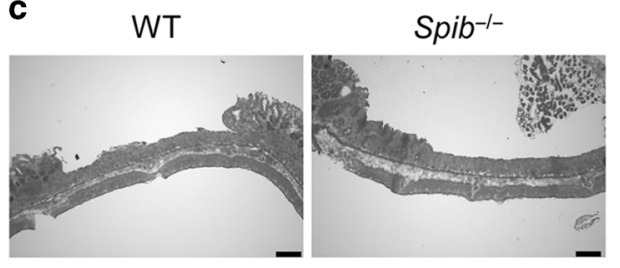

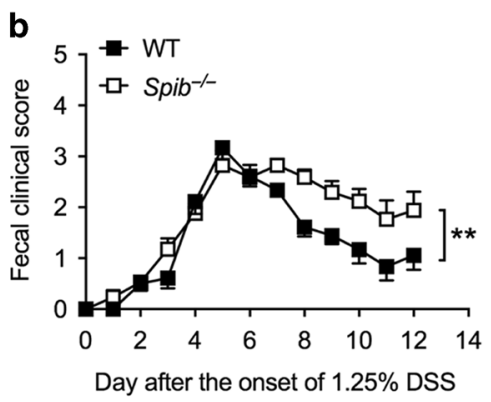

d

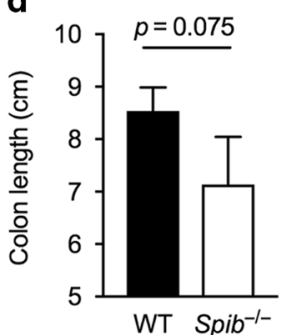

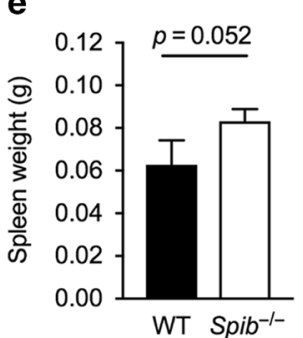

Fig. 6 Susceptibility of Spib $^{-/-}$mice to DSS-induced colitis. a-e WT or Spib ${ }^{-/-}$mice received $1.25 \%$ (a-d) or $0.75 \%$ (e) DSS in drinking water for 7 days followed by normal water. Body weights (a) and clinical scores (b) were measured every day during the experimental period. The data are shown as the mean \pm SEM $(n=18,17)$ from two independent experiments; ${ }^{*} p<0.05,{ }^{*} p<0.01$ by two-way ANOVA testing. c Representative histological images of the middle parts of colon of WT and Spib ${ }^{-/-}$mice at day 12 are shown. Scale bar, $300 \mu \mathrm{m}$. d Colon lengths were measured at day 12 after beginning DSS treatment. e Spleen weights were measured at day 7 after the beginning of $0.75 \%$ DSS treatment. d, e The data are shown as the mean $\pm \operatorname{SD}(n=3) .{ }^{*} p<0.05,{ }^{* *} p<0.01$ by Student's $t$ test. 
a

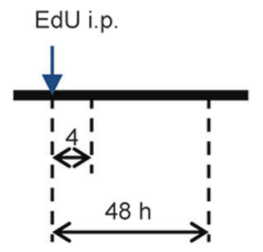

b

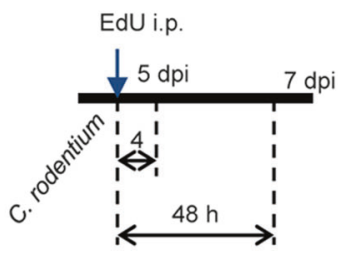

C

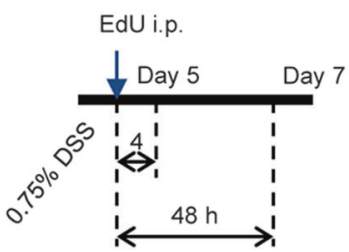

WT

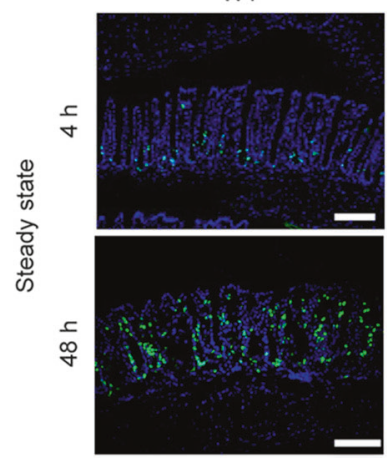

WT

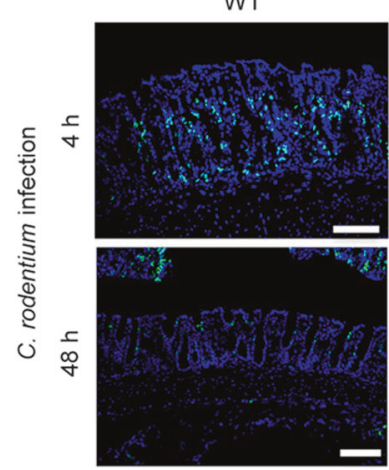

WT

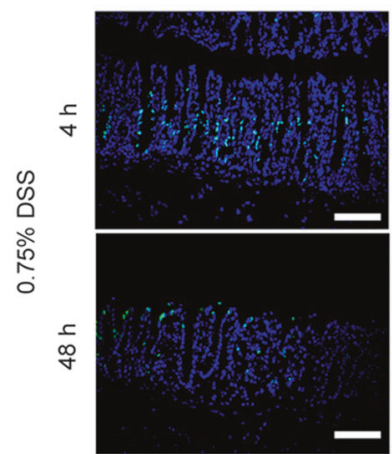

Spib-/-

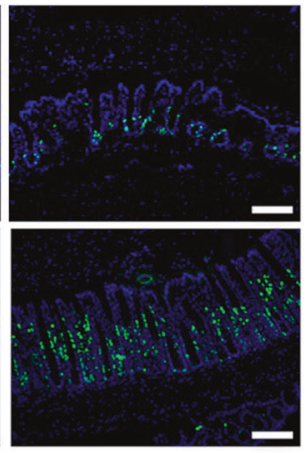

Spib-/-

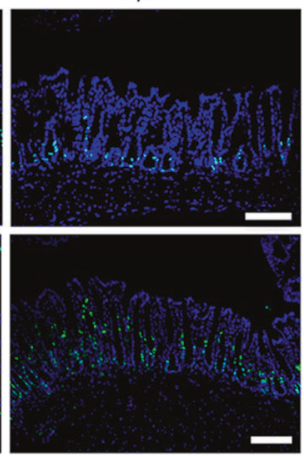

Spib ${ }^{-1-}$

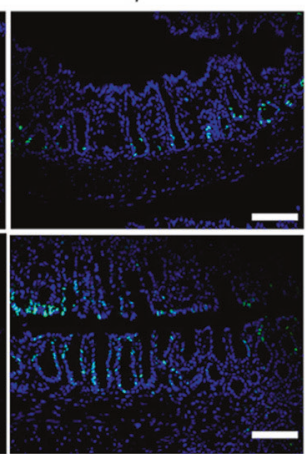

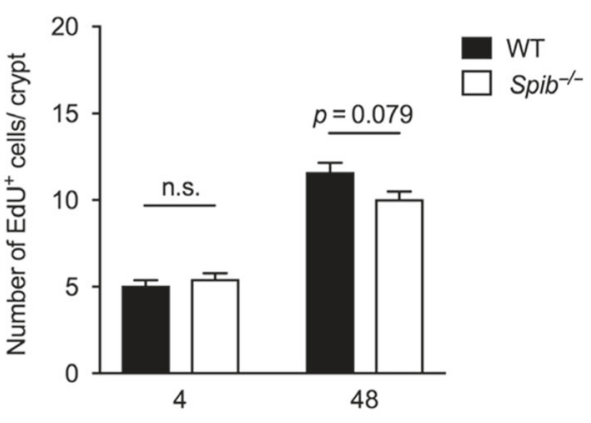

h

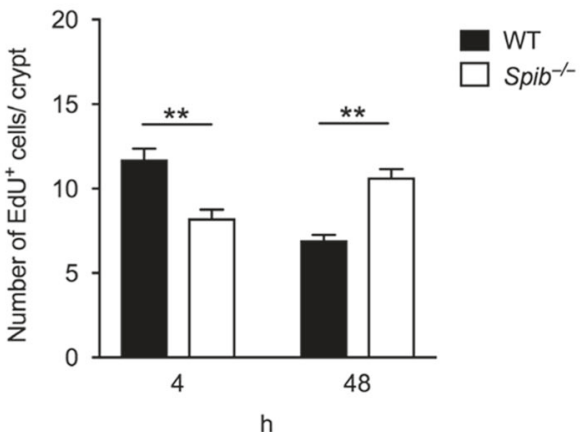

h

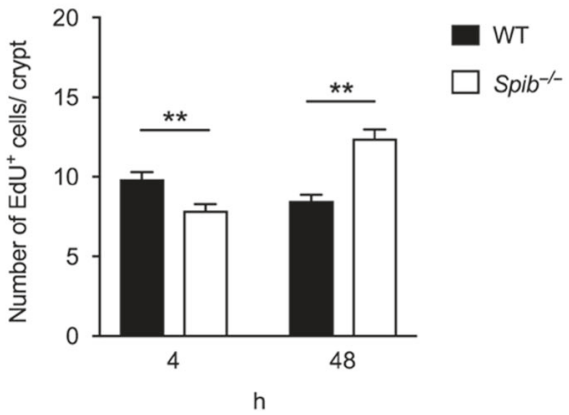

Fig. 7 The acceleration of epithelial cell turnover was inhibited in Spib ${ }^{-1-}$ mice. a, b EdU was intraperitoneally injected into WT and Spib ${ }^{-/-}$ mice under noninfected (a) or infected (b) conditions. c At day 5 after the onset of $0.75 \%$ DSS drinking, EdU was intraperitoneally injected into the mice. EdU ${ }^{+}$cells were visualized with Alexa-Flour 488 at 4 and $48 \mathrm{~h}$ after EdU injection. Fifteen crypts were selected at random to calculate $\mathrm{EdU}^{+}$cells $(4 \mathrm{~h}, n=90$ [a-c] from 6 mice; $48 \mathrm{~h}, n=105$ [a, b] or 120 [c] from 7 mice). Nucleus was stained by Hoechst 33342 . Scale bars, $100 \mu \mathrm{m}$. The data are shown as the mean \pm SEM from two independent experiments; ${ }^{*} p<0.05,{ }^{* *} p<0.01$ by two-way ANOVA testing.

$\mathrm{Spib}^{-/-}$mice (Fig. 7c; $4 \mathrm{~h}$ postinjection), whereas cell turnover was significantly delayed (Fig. 7c; $48 \mathrm{~h}$ postinjection). Together, these observations illustrated that induction of commensal-specific immune responses by $M$ cell-dependent antigen uptake may facilitate vigorous epithelial cell turnover in response to infection and inflammatory stimuli, which may reinforce mucosal barrier functions (Supplementary Fig. 12).

\section{DISCUSSION}

The lack of mature $M$ cells increased susceptibility to $C$. rodentium infection and exacerbated infectious colitis and systemic bacterial translocation. Our BM chimera experiments revealed that nonhematopoietic, but not hematopoietic, Spi-B was essential for ameliorating $C$. rodentium-induced colitis. While hematopoietic Spi-B is expressed in plasmacytoid DCs, B cells, and common myeloid cell progenitors, ${ }^{27-29}$ non-hematopoietic Spi-B is predominantly expressed in $M$ cells. ${ }^{24,37}$ Hence, $M$ cells contribute to preventing severe colitis induced by $C$. rodentium infection.

The deletion of $B$ cells can cause persistent infection of $C$. rodentium. ${ }^{19,38}$ In addition, $C$. rodentium-specific serum $\mathrm{lgG}$, but not fecal $\operatorname{lgA}$, was required for clearance of the pathogen. ${ }^{18,38,39}$ We also observed that $C$. rodentium was eradicated in both WT and $\mathrm{Spib}^{-1-}$ mice following $C$. rodentium-specific serum IgG induction. In contrast, pathogen-specific fecal $\lg A$ induction was impaired only in Spib ${ }^{-/-}$mice. These results indicate that $M$ cellmediated antigen transport was vital for $C$. rodentium-specific $\lg A$ responses but dispensable for inducing $\lg G$ responses needed to eradicate the pathogen.

$\mathrm{CD}^{+} \mathrm{T}$ cells represent another essential element for host defense against $C$. rodentium infection. ${ }^{19,39}$ Our time-course analysis showed that $C$. rodentium-specific Th1, Th17, and Th22 
cells were not detected in the CLP of WT mice until late-stage infection (16 dpi). Unexpectedly, M cell deficiency did not affect the pathogen-specific Th17 response and even enhanced Th1 and Th22 responses in the effector site during late-stage infection. Nevertheless, such cellular immune responses were only minimally induced in the GALTs of Spib ${ }^{-1-}$ mice during infection, indicating that pathogen-specific Th1, Th17, and Th22 responses were induced in the colon and/or systemic lymphoid tissue, such as the spleen. During $C$. rodentium infection, defects in mucosal barrier function enhanced the systemic translocation of opportunistic bacteria in addition to C. rodentium. ${ }^{23,40,41}$ Similarly, Spi-B deficiency augmented the systemic translocation of luminal bacteria during infection, indicative of attenuated barrier functions. We also observed a remarkable increase in $C$. rodentiumspecific Th1 cells in the spleen of Spib ${ }^{-/-}$mice, suggesting that the massive bacterial burden during the early stage induced potent $C$. rodentium-specific Th1 responses in systemic lymphoid tissues of $\mathrm{Spib}^{-1-}$ mice during the late stage.

Several types of antigen-uptake machineries other than the $M$ cell-dependent pathways have been defined. $\mathrm{CX}_{3} \mathrm{CR} 1^{+}$mononuclear phagocytes protrude their transepithelial dendrites to directly sample luminal antigens and facilitate the development of regulatory $T$ cells that induce oral tolerance to ovalbumin. ${ }^{42}$ However, our data demonstrated that $\mathrm{M}$ cell-dependent antigen uptake played a non-redundant role in establishing the gut immune system. In GALTs, M cells vigorously internalize soluble antigens and microorganisms and transport them to underlying immature DCs. ${ }^{6,43}$ This process provokes GC reactions and establishes Th cell memory for commensal microbes, which is critical for limiting the dissemination of commensals during mucosal infection. Activated Th1 cells abundantly produce IFN- $\gamma$ to activate macrophages and promote epithelial turnover. ${ }^{20,21}$ In addition, Th17 cells can express IL-17A to activate neutrophils. Th22-derived IL-22 upregulates antimicrobial peptides in epithelial cells and promotes intestinal stem cell proliferation. ${ }^{44}$ Therefore, $\mathrm{T}$ cell-deficient mice are highly susceptible to infectious diseases and often succumb to sublethal mucosal pathogens. ${ }^{19,39}$ Although little is known regarding the contribution of GALTs to the induction of cellular immunity, our data demonstrated that GALTs are a primary site for inducing commensal-specific Th1, Th17, and Th22 responses. Thus M celldependent antigen uptake is critical for immune surveillance on the mucosal surface.

The barrier dysfunction in Spib ${ }^{-/-}$mice likely resulted from defects in commensal-specific immune responses during earlystage infection. The absence of $M$ cells due to Spi-B deficiency caused hypoplasia of GALTs with prominent decreases in Th1, Th17, and Th22 cells, as well as GC B cells under physiological conditions. Accordingly, development and/or maintenance of central and resident memory $\mathrm{CD}^{+} \mathrm{T}$ cells were severely affected in the absence of $M$ cells. Consistent with these observations, commensal-specific cellular immunity, especially Th1 and Th22 responses, were severely affected in the effector site of Spib ${ }^{-/-}$ mice until middle-stage infection (0-12 dpi). In addition, Spib ${ }^{-/-}$ mice showed increased susceptibility to DSS-induced noninfectious colitis. These abnormalities were associated with attenuated proliferative response in colonic epithelial cells, which serves as a fundamental barrier to repair injured cells during mucosal infection and inflammation. ${ }^{36}$ These results suggest that preexisting commensal-specific Th cell memory induced by $M$ celldependent antigen transport may be essential for establishing the mucosal barrier to prevent colitis, although further investigations are necessary to directly test this possibility. It is also possible to speculate that commensal-specific effector Th cells may have crossreacted $C$. rodentium-derived antigens analogous to commensal bacterial antigens and limit persistent colonization of $C$. rodentium in WT, but not in $\mathrm{Spib}^{-1-}$, mice. However, this speculation is unlikely, because $C$. rodentium-specific Th cells are nearly absent until middle-stage infection in both groups and even more abundant in the CLP of Spib ${ }^{-/-}$mice than WT mice at late-stage infection (Fig. 3d).

Accumulating evidence has implicated commensal microbes in the development of autoimmune and allergic diseases. ${ }^{45-48}$ For instance, commensal gut-segmented filamentous bacteria induce follicular helper T (Tfh) cells in PP. Gut-derived Tfh cells migrate to the systemic lymphoid tissues to promote autoantibody production and arthritis development. ${ }^{49}$ This raises the possibility that $\mathrm{M}$ cell-dependent uptake of commensal microbes may significantly promote the pathogenesis of autoimmune diseases. However, human IgA deficiency has also been associated with the development of autoimmune diseases and food allergies. ${ }^{50,51}$ Loss of the S-IgA-mediated mucosal barrier may increase intestinal permeability, which could cause systemic inflammation and allergic responses by compromising oral tolerance. Studying $M$ cell-null mice in allergic and autoimmune disease models would uncover the covert role of $\mathrm{M}$ cell-dependent antigen uptake in the development of immune disorders.

\section{METHODS}

Animal experiments

Spib $^{-1-}$ mice in a C57BL/6 background ${ }^{27}$ and Ly5.1 congenic C57BL/6J mice ${ }^{52}$ were bred and maintained under conventional (CV) conditions at an animal facility at the Institute of Medical Science, The University of Tokyo (IMSUT) and under specific pathogen-free conditions at an animal facility at Keio University. To examine the influence of distinct commensal bacteria, Spib ${ }^{-1-}$ mice and age-matched C57BL/6J mice were purchased from CLEA Japan, Inc. (Tokyo, Japan), co-housed for at least 3 weeks, and examined under CV conditions. Protocols for all experiments were approved by the relevant animal study committees of IMSUT and Keio University.

\section{C. rodentium infection}

C. rodentium DBS100 was provided by $\mathrm{H}$. Mimuro. Male and female mice were orally infected with $10^{9}$ colony-forming units (cfu) of $C$. rodentium in Luria-Bertani broth. Bacterial loads in feces were determined by performing cfu assays using MacConkey agar (Merck, Darmstadt, Germany) with 1\% lactose. The detection limit of the assay was $10^{3} \mathrm{cfu} / \mathrm{mg}$ feces.

Total fecal clinical scores (0-5) were assessed by summing individual diarrhea and blood scores, as follows. Scores related to diarrhea were 0 (normal), 1 (watery stool without deformation), 2 (watery stool with deformation), and 3 (diarrhea). Scores related to blood in the stool were 0 (normal), 1 (partial bloody stool), and 2 (bloody stool).

\section{Histology}

For hematoxylin and eosin staining, colons were fixed using Zinc Formalin (Wako Pure Chemical Industries, Osaka, Japan) for $4 \mathrm{~h}$ and paraffin embedded. Histological severity was scored based on three criteria: edema (0-3), disruption of epithelial integrity (0-4), and cell infiltration (0-3). Edema was scored as 0 (normal), 1 $(25-50 \%), 2(50-75 \%)$, or 3 ( $>75 \%$ damaged lamina propria). Disruption of epithelial integrity was scored as 0 (normal), 1 (10\% epithelial cell shedding), or 2 ( $20 \%$ epithelial cell shedding). A score of 3 was assigned if ulceration was observed or a score of 4 was assigned if broad crypt destruction was noted. Cell infiltration was scored as 0 (none), 1 (mild), 2 (moderate), or 3 (severe).

Bacterial translocation into the spleen

After weighing, each spleen was homogenized in sterile phosphate-buffered saline (PBS) and centrifuged at $500 \mathrm{rpm}$ for $30 \mathrm{~s}$ to remove host cells. The supernatants were cultured on Difco $^{\mathrm{TM}}$ Brain Heart Infusion Ager (BD, MD, USA) at $37^{\circ} \mathrm{C}$ under aerobic or anaerobic conditions. 
BM chimera experiment

BM cells $\left(1 \times 10^{7}\right)$ were transferred sublethally into 12-Gyirradiated recipient 8 -week-old mice via the orbital vein. Chimeras were additionally maintained for 4-6 weeks under CV conditions. The mice were treated with $0.5 \mathrm{mg} / \mathrm{ml}$ ampicillin and $1 \mathrm{mg} / \mathrm{ml}$ neomycin in drinking water to prevent intestinal inflammation for 2 weeks. The efficiency of BM transplantation was confirmed by flow cytometric analysis of white blood cells, based on CD45.1 and CD45.2 expression at 6 weeks post-transfer; mice whose blood lymphocytes were dominantly occupied by donor-derived hematopoietic cells (approximately 70-90\%) were used for infection studies.

Enzyme-linked immunosorbent assay (ELISA) experiments Feces collected from Spib ${ }^{-/-}$mice and co-housed WT mice were lyophilized and suspended in PBS containing a Complete proteinase inhibitor cocktail (Roche, Mannheim, Germany). The supernatants of homogenized feces were subjected to an $\lg A$ ELISA using a mouse IgA ELISA quantitation set (Bethyl Laboratories, Inc., TX, USA), following the manufacturer's instructions.

\section{Leukocyte preparation}

Single-cell suspensions of gut tissues and $\mathrm{mLNs}$ were prepared as described. ${ }^{53}$ Briefly, colonic tissue specimens were magnetically stirred in Hanks' balanced salt solution containing $30 \mathrm{mM}$ EDTA.2Na, $1 \mathrm{mM}$ dithiothreitol, and $12.5 \mathrm{mM}$ HEPES ( $\mathrm{pH} 7.3$ ) to remove the colonic epithelium. The remaining colonic tissue, ileal PPs (three samples upward from the terminal of the ileum), and cecal patches were minced and stirred at $37^{\circ} \mathrm{C}$ for $30 \mathrm{~min}$ in dissociation buffer, comprised of RPMI 1640 medium supplemented with $0.5 \mathrm{mg} / \mathrm{ml}$ collagenase (Wako Pure Chemical), $0.5 \mathrm{mg} / \mathrm{ml}$ DNase I (Wako), 100 units $/ \mathrm{ml}$ penicillin, $100 \mu \mathrm{g} / \mathrm{ml}$ streptomycin, $12.5 \mathrm{mM}$ HEPES (pH 7.3), and $2 \%$ fetal bovine serum (FBS). The $\mathrm{mLNs}$ were homogenized on a $100-\mu \mathrm{m}$ cell strainer (Grainer BioOne, Austria). The spleen was injected with dissolution buffer, incubated at room temperature for $30 \mathrm{~min}$, and homogenized on a $100-\mu \mathrm{m}$ cell strainer (Grainer). The obtained single-cell suspensions were analyzed by flow cytometry or cultured after purifying the leukocytes by Percoll-gradient separation. ${ }^{53}$

\section{Flow cytometry}

Antibodies conjugated with fluorescein isothiocyanate (FITC), phycoerythrin, PerCP-Cy5.5, PerCP-eFluor710, PE-Cy7, allophycocyanin (APC), redFlour710, APC-eFlour780, APC-H7, V421, V500, or biotin were purchased from BD Biosciences, Thermo Fisher Scientific, BioLegend, or Tonbo Biosciences. Cells were incubated with a TruStain $\mathrm{fCX}^{\mathrm{TM}}$ (anti-mouse CD16/CD32) antibody (101320, BioLegend) to block non-specific staining, following by incubation with antibodies against CD3ع, CD4, CD8a, CD11b, CD11c, CD44, CD45, CD45.1, CD45.2, CD45R (B220), CD62L, CD69, CD95, GL7, IgA, Ly-6G/Ly-6C(Gr1), KLRG1, or MHC class II (Supplemental Table 1). For intracellular staining of transcription factors, cells were fixed in Fixation/Permeabilization Diluent (Thermo Fisher Scientific) after cell-surface staining and labeling dead cells with the LIVE/DEAD ${ }^{\text {TM }}$ Fixable Aqua Dead Cell Stain Kit (Life Technologies, CA, USA). The fixed cells were incubated with antibodies against Foxp3, Gata3, ROR-yt, and T-bet in Permeabilization Buffer (Thermo Fisher Scientific). For intracellular cytokine staining, primary lymphocytes were cultured in RPMI1640 containing GlutaMAX ${ }^{T M}-1,100$ units $/ \mathrm{ml}^{2}$ penicillin, $100 \mu \mathrm{g} / \mathrm{ml}$ streptomycin, $12.5 \mathrm{mM}$ HEPES, 10\% FBS, phorbol 12-myristate 13-acetate, and ionomycin (Sigma-Aldrich) at $37^{\circ} \mathrm{C}$ for $30 \mathrm{~min}$, and the cells were further cultured for $4 \mathrm{~h}$ in the presence of $10 \mu \mathrm{g} / \mathrm{ml}$ Brefeldin $A$ (BFA; Sigma-Aldrich) to block cytokine secretion. The cultured cells were fixed in Cytofix/CytoPerm (BD Biosciences) after cellsurface staining and staining dead cells. Next, the cells were stained with antibodies against IFN- $\gamma$, IL-17A, and IL-22 in Perm
Wash buffer (BD Biosciences). Flow cytometric analysis was performed using a FACSAria III or LSR II instrument (BD Biosciences), and the data were analyzed using the FlowJo software (Tree Star, Inc., RRID: SCR_008520).

Detection of antigen-specific Th cells

Splenic DCs were enriched with the iMag sorting system (BD Biosciences) using a biotin-conjugated anti-CD11c antibody (BioLegend) and streptavidin particles plus (BD Biosciences). DCs were incubated with antigens, namely, heat-killed $C$. rodentium or fecal suspensions, and cultured overnight in RPMI 1640 medium containing $10 \%$ FBS, GlutaMAX ${ }^{T M}$ (Life Technologies), 100 units $/ \mathrm{ml}$ penicillin, $100 \mathrm{mg} / \mathrm{ml}$ streptomycin, and $12.5 \mathrm{mM}$ HEPES. Lymphocytes from various tissues were co-cultured with antigen-pulsed splenic DCs and additional antigens for $2 \mathrm{~h}$ and then cultured in the presence of $10 \mu \mathrm{g} / \mathrm{ml}$ BFA for $4 \mathrm{~h}$. As a negative reference, lymphocytes were cultured with antigen-unloaded splenic DCs. The background negative reference values were subtracted from those of co-cultured lymphocytes and antigen-pulsed DCs to determine the number of antigen-specific Th cells.

IgA-bacterial flow cytometry

IgA-bacterial flow cytometry was performed as described. ${ }^{54}$ Briefly, mouse feces were homogenized in a $70-\mu \mathrm{m}$ cell strainer with sterile PBS. After brief centrifugation at $500 \times g$ for $5 \mathrm{~min}$ to remove contaminants, bacteria were stained with an FITCconjugated anti-lgA (BD Biosciences) antibody and 4,6-diamidino-2-phenylindole to detect live bacteria. Flow cytometric analysis was performed with an FACSAria III instrument (BD Biosciences). The data obtained were analyzed using the FlowJo software.

\section{DSS-induced colitis model}

Mice received 1.25 or $0.75 \%$ DSS (molecular weight: 36,000-50,000, MP Biomedicals, Santa Ana, CA, USA) in drinking water for 7 days, followed by regular water for 5 days. Body weight, water consumption, and clinical scores were monitored daily. Clinical scores were determined as described for the $C$. rodentium infection model.

In vivo EdU incorporation assay

WT and Spib ${ }^{-/-}$mice were killed $4 \mathrm{~h}$ or 2 days after intraperitoneal injection of $1 \mathrm{mg}$ EdU in PBS, and colons were embedded in O.C.T. compound. Slices were fixed in Mildform 10NM (Wako) for $15 \mathrm{~min}$ and permeabilized with $0.5 \%$ Triton X-100 in PBS for 20 min. EdU was detected using Click-iT EdU Imaging Kits (Invitrogen) following the manufacturer's instructions.

\section{Statistical analysis}

Differences between two groups were analyzed with Student's $t$ test or Welch's $t$ test in the case of homoscedasticity or heteroscedasticity, respectively. When normality was not found in Shapiro-Wilk test, the data were analyzed with Mann-Whitney $U$ test. Body weight changes, fecal clinical scores, and fecal cfu in $\mathrm{Spib}^{-/-}$mice were analyzed by repeated-measures analysis of variance testing followed by Tukey's test. $p$ Values of $<0.05$ were defined as statistically significant.

\section{ACKNOWLEDGEMENTS}

We would like to thank Yuuki Obata, Chiaki Yarimizu, Shun Murayama, Yu Miyamoto, Takahiro Yamada (Keio University), and Naoki Takemura (IMSUT, Chiba University) for providing technical support. We also thank Yun-Gi Kim (Keio University) for valuable discussions. This study was supported by funding from MEXT and JSPS Grants-in-Aid for Scientific Research (grants 17H04089, 16H01369, 25293114, and 26116709 [to K.H.]; grant 16J05903 [to Y.N.]; grant 17K19551 [to H.M.]; grant 18H02674 [to J.K.]; and grant $18 \mathrm{H} 05280$ [to H.K.]), the Japan Agency for Medical Research and Development (AMED) (grants 16gm1010004h0101, 17gm1010004h0102, and 18gm1010004h0103 [to K.H.] 
and grants 18gm1010006h0002, 18ak0101068h0002, and 18ek0410032s0103 [to J.K.]), The Takeda Science Foundation (to K.H.), The Daiichi Sankyo Foundation of Life Science (to K.H.), The Terumo Foundation for Life Science and Arts (to K.H. and J.K.), The Canon Foundation (to J.K.), The Asahi Glass Foundation (to K.H.), The Naito Foundation (to H.M.), the Grant for Joint Research Project of the Institute of Medical Science, the University of Tokyo (to K.H.), and The Keio University Special Grant-in-Aid for Innovative Collaborative Research Projects (to K.H.).

\section{AUTHOR CONTRIBUTIONS}

Y.N. and K.H. conceived of the study and wrote the manuscript. Y.N. entirely performed the study design, experiments, and data analysis. Y. Furusawa and D.T. participated in some of experiments. Y. Fujimura provided support for the histological analysis. H.M., J.K., T.K., and H.K. provided experimental resources. K.H. organized the study and the interpreted data. All authors reviewed the manuscript critically and approved the final version.

\section{ADDITIONAL INFORMATION}

The online version of this article (https://doi.org/10.1038/s41385-020-0263-0) contains supplementary material, which is available to authorized users.

Competing interests: The authors declare no competing interests.

Publisher's note Springer Nature remains neutral with regard to jurisdictional claims in published maps and institutional affiliations.

\section{REFERENCES}

1. Croxen, M. A. et al. Recent advances in understanding enteric pathogenic Escherichia coli. Clin. Microbiol. Rev. 26, 822-880 (2013).

2. Lima, I. F. N., Havt, A. \& Lima, A. A. M. Update on molecular epidemiology of Shigella infection. Curr. Opin. Gastroenterol. 31, 30-37 (2015).

3. Talavera, A. \& Pérez, E. M. Is cholera disease associated with poverty? J. Infect. Dev. Ctries. 3, 408-411 (2009).

4. Neutra, M. R., Pringault, E. \& Kraehenbuhl, J. P. Antigen sampling across epithelial barriers and induction of mucosal immune responses. Annu. Rev. Immunol. 14, 275-300 (1996).

5. Owen, R. L. \& Jones, A. L. Epithelial cell specialization within human Peyer's patches: an ultrastructural study of intestinal lymphoid follicles. Gastroenterology 66, 189-203 (1974)

6. Bockman, D. E. \& Cooper, M. D. Pinocytosis by epithelium associated with lymphoid follicles in the bursa of Fabricius, appendix, and Peyer's patches. An electron microscopic study. Am. J. Anat. 136, 455-477 (1973).

7. Nakamura, Y., Kimura, S. \& Hase, K. M cell-dependent antigen uptake on follicleassociated epithelium for mucosal immune surveillance. Inflamm. Regen. 38, 15 (2018).

8. Hase, K. et al. Uptake through glycoprotein 2 of $\mathrm{FimH}(+)$ bacteria by $\mathrm{M}$ cells initiates mucosal immune response. Nature 462, 226-230 (2009).

9. Shima, H. et al. A novel mucosal vaccine targeting Peyer's patch $M$ cells induces protective antigen-specific IgA responses. Int. Immunol. 26, 619-625 (2014).

10. Matsumura, T. et al. Botulinum toxin A complex exploits intestinal $M$ cells to enter the host and exert neurotoxicity. Nat. Commun. 6, 6255 (2015).

11. Amerongen, H. M., Wilson, G. A., Fields, B. N. \& Neutra, M. R. Proteolytic processing of reovirus is required for adherence to intestinal M cells. J. Virol. $\mathbf{6 8}, \mathbf{8 4 2 8 - 8 4 3 2}$ (1994).

12. Autenrieth, I. B. \& Firsching, R. Penetration of $M$ cells and destruction of Peyer's patches by Yersinia enterocolitica: an ultrastructural and histological study. J. Med. Microbiol. 44, 285-294 (1996).

13. Nakato, G. et al. Cutting edge: Brucella abortus exploits a cellular prion protein on intestinal M cells as an invasive receptor. J. Immunol. 189, 1540-1544 (2012).

14. Sansonetti, P. J., Arondel, J., Cantey, J. R., Prévost, M. C. \& Huerre, M. Infection of rabbit Peyer's patches by Shigella flexneri: effect of adhesive or invasive bacterial phenotypes on follicle-associated epithelium. Infect. Immun. 64, 2752-2764 (1996).

15. Takakura, l. et al. Orally administered prion protein is incorporated by $\mathrm{m}$ cells and spreads into lymphoid tissues with macrophages in prion protein knockout mice. Am. J. Pathol. 179, 1301-1309 (2011).

16. Fuglem, B. et al. Antigen-sampling cells in the salmonid intestinal epithelium. Dev. Comp. Immunol. 34, 768-774 (2010).

17. Mundy, R., MacDonald, T. T., Dougan, G., Frankel, G. \& Wiles, S. Citrobacter rodentium of mice and man. Cell. Microbiol. 7, 1697-1706 (2005).
18. Kamada, N. et al. Humoral immunity in the gut selectively targets phenotypically virulent attaching-and-effacing bacteria for intraluminal elimination. Cell Host Microbe 17, 617-627 (2015).

19. Simmons, C. P. et al. Central role for B lymphocytes and CD4+ T cells in immunity to infection by the attaching and effacing pathogen Citrobacter rodentium. Infect. Immun. 71, 5077-5086 (2003).

20. Shiomi, H. et al. Gamma interferon produced by antigen-specific CD4+ T cells regulates the mucosal immune responses to Citrobacter rodentium infection. Infect. Immun. 78, 2653-2666 (2010).

21. Chan, J. M. et al. CD4+ T cells drive goblet cell depletion during Citrobacter rodentium infection. Infect. Immun. 81, 4649-4658 (2013).

22. Basu, R. et al. Th22 cells are an important source of IL-22 for host protection against enteropathogenic bacteria. Immunity 37, 1061-1075 (2012).

23. Pham, T. A. N. et al. Epithelial IL-22RA1-mediated fucosylation promotes intestinal colonization resistance to an opportunistic pathogen. Cell Host Microbe 16 504-516 (2014).

24. Kanaya, T. et al. The Ets transcription factor Spi-B is essential for the differentiation of intestinal microfold cells. Nat. Immunol. 13, 729-736 (2012).

25. Atarashi, K. et al. Th17 cell induction by adhesion of microbes to intestinal epithelial cells. Cell 163, 367-380 (2015).

26. Spahn, T. W. et al. CD4+ T cells transfer resistance against Citrobacter rodentiuminduced infectious colitis by induction of Th 1 immunity. Scand. J. Immunol. 67, 238-244 (2008).

27. Sasaki, I. et al. Spi-B is critical for plasmacytoid dendritic cell function and development. Blood 120, 4733-4743 (2012).

28. Su, G. H. et al. Defective B cell receptor-mediated responses in mice lacking the Ets protein, Spi-B. EMBO J. 16, 7118-7129 (1997).

29. Shimokawa, C. et al. Mast cells are crucial for induction of group 2 innate lymphoid cells and clearance of helminth infections. Immunity 46, 863-874.e4 (2017).

30. Shin, H. \& Iwasaki, A. Tissue-resident memory T cells. Immunol. Rev. https://doi. org/10.1111/imr.12087 (2013)

31. Baptista, A. P. et al. Colonic patch and colonic SILT development are independent and differentially regulated events. Mucosal Immunol. 6, 511-521 (2013).

32. Adachi, S., Yoshida, H., Kataoka, H. \& Nishikawa, S. Three distinctive steps in Peyer's patch formation of murine embryo. Int. Immunol. 9, 507-514 (1997).

33. Ishigame, $H$. et al. Differential roles of interleukin-17A and $-17 F$ in host defense against mucoepithelial bacterial infection and allergic responses. Immunity $\mathbf{3 0}$ 108-119 (2009).

34. Zheng, Y. et al. Interleukin-22 mediates early host defense against attaching and effacing bacterial pathogens. Nat. Med. 14, 282-289 (2008).

35. Gibson, D. L. et al. MyD88 signalling plays a critical role in host defence by controlling pathogen burden and promoting epithelial cell homeostasis during Citrobacter rodentium-induced colitis. Cell. Microbiol. 10, 618-631 (2008).

36. Kim, M. et al. Bacterial interactions with the host epithelium. Cell Host Microbe 8 20-35 (2010).

37. Sato, S. et al. Transcription factor Spi-B-dependent and -independent pathways for the development of Peyer's patch M cells. Mucosal Immunol. 6, 838-846 (2013).

38. Maaser, C. et al. Clearance of Citrobacter rodentium requires B cells but not secretory immunoglobulin $\mathrm{A}(\lg \mathrm{A})$ or $\lg \mathrm{M}$ antibodies. Infect. Immun. 72, 3315-3324 (2004).

39. Bry, L. \& Brenner, M. B. Critical role of T cell-dependent serum antibody, but not the gut-associated lymphoid tissue, for surviving acute mucosal infection with Citrobacter rodentium, an attaching and effacing pathogen. J. Immunol. 172, 433-441 (2004).

40. Flynn, A. N. \& Buret, A. G. Tight junctional disruption and apoptosis in an in vitro model of Citrobacter rodentium infection. Microb. Pathog. 45, 98-104 (2008).

41. Vallance, B. A., Deng, W., Jacobson, K. \& Finlay, B. B. Host susceptibility to the attaching and effacing bacterial pathogen Citrobacter rodentium. Infect. Immun. 71, 3443-3453 (2003).

42. Mazzini, E., Massimiliano, L., Penna, G. \& Rescigno, M. Oral tolerance can be established via gap junction transfer of fed antigens from $\mathrm{CX} 3 \mathrm{CR}^{+}$macrophages to $\mathrm{CD}_{103^{+}}$dendritic cells. Immunity 40, 248-261 (2014).

43. Neutra, M. R., Phillips, T. L., Mayer, E. L. \& Fishkind, D. J. Transport of membranebound macromolecules by $M$ cells in follicle-associated epithelium of rabbit Peyer's patch. Cell Tissue Res. 247, 537-546 (1987).

44. Lindemans, C. A. et al. Interleukin-22 promotes intestinal-stem-cell-mediated epithelial regeneration. Nature 528, 560-564 (2015).

45. Berer, K. et al. Commensal microbiota and myelin autoantigen cooperate to trigger autoimmune demyelination. Nature 479, 538-541 (2011).

46. Vatanen, $T$. et al. The human gut microbiome in early-onset type 1 diabetes from the TEDDY study. Nature 562, 589-594 (2018). 
Microfold cell-dependent antigen transport alleviates infectious colitis...

Y Nakamura et al.

690

47. Horai, R. et al. Microbiota-dependent activation of an autoreactive T cell receptor provokes autoimmunity in an immunologically privileged site. Immunity 43, 343-353 (2015).

48. McKenzie, C., Tan, J., Macia, L. \& Mackay, C. R. The nutrition-gut microbiomephysiology axis and allergic diseases. Immunol. Rev. 278, 277-295 (2017).

49. Teng, F. et al. Gut microbiota drive autoimmune arthritis by promoting differentiation and migration of Peyer's patch T follicular helper cells. Immunity 44, 875-888 (2016).

50. Aghamohammadi, A. et al. IgA deficiency: correlation between clinical and immunological phenotypes. J. Clin. Immunol. 29, 130-136 (2009).
51. Fadlallah, J. et al. Microbial ecology perturbation in human IgA deficiency. Sci. Transl. Med. 10, eaan1217 (2018).

52. Komatsu, N. et al. Heterogeneity of natural Foxp3+ T cells: a committed regulatory T-cell lineage and an uncommitted minor population retaining plasticity. Proc. Natl Acad. Sci. USA 106, 1903-1908 (2009).

53. Obata, Y. et al. Epithelial-stromal interaction via Notch signaling is essential for the full maturation of gut-associated lymphoid tissues. EMBO Rep. 15, 1297-1304 (2014).

54. Nagashima, K. et al. Identification of subepithelial mesenchymal cells that induce IgA and diversify gut microbiota. Nat. Immunol. 18, 675-682 (2017). 